\title{
Camera Sensory System for Door and Stair Detection for Visually Impaired
}

\author{
Shrugal Varde, M.S. Panse
}

\begin{abstract}
This paper introduces a novel travel for blind users that can assist them to detects location of doors in corridors and also give information about location of stairs. The developed system uses camera to capture images in front of the user. Feature extraction algorithm is used to extract key features that distinguish doors and stairs from other structures observed in indoor environments. This information is then conveyed to the user using simple auditory feedback. The mobility aid was validated on 50 visually impaired users. The subjects walked in a controlled test environment. The accuracy of the device to help the user detect doors and stairs was determined. The results obtained were satisfactory and the device has the potential for use in standalone mode for indoor navigations.
\end{abstract}

Keywords: Visually impaired, stereo, mobility, doors, stairs navigation

\section{INTRODUCTION}

Vision impairment means anatomic and functional disturbances of the sense of vision of sufficient magnitude to cause total loss of light perception. A person is said to have low vision when their eyesight is limited or impaired and cannot be adequately corrected with surgery, conventional glasses, or contact lenses. Low vision is measured by distance visual acuity [2]. When a person has a normal vision, their visual acuity is rated $6 / 6$ or $20 / 20$. This rating indicates that a person who has a normal vision can read the letters on an eye chart which is designed to be seen at a distance of six meters or twenty feet away. A person is said to have low vision when they see fewer letters on the eye chart from this distance. For example, $6 / 18$ means that the patient can see at 6 meters what a person with normal vision can see at 18 meters.

World Health Organization (WHO) surveyed in 2010 to estimate the total number of visually impaired (VI) people in the world [1]. Numbers clearly indicate that there is a need of advanced mobility aid that can help visually impaired used in their day-to-day needs.

Electronic travel aids are devices that transform information about the environment that would normally be relayed through vision into a form that can be conveyed

Manuscript received on August 07, 2021.

Revised Manuscript received on August 14, 2021.

Manuscript published on August 30, 2021.

* Correspondence Author

Shrugal Varde*, Pursuing, Ph.D. degree, Department of Electronic Engineering, Veermata Jijabai Technological Institute (VJTI.) Mumbai (Maharashtra) India.

Dr. M.S. Panse, Professor, Department of Electronic Engineering, Veermata Jijabai Technological Institute (VJTI.) Mumbai (Maharashtra) India.

(c) The Authors. Published by Blue Eyes Intelligence Engineering and Sciences Publication (BEIESP). This is an open access article under the CC BY-NC-ND license (http://creativecommons.org/licenses/by-nc-nd/4.0/) through another sensory modality The National Research Council provided guidelines that should be considered while designing an ETA [4,5]. They are as follows:

1. obstacles in the travel path from ground level to head height for the full body width should be detected.

2. Travel surface information including textures and discontinuities.

3. Detection of objects bordering the travel path for shore lining and projection.

4. Landmark location and identification information.

5. The mobility aid should be a single unit, reliable, have low power consumption and cosmetically acceptable.

6. The device should operate with minimum interference to natural sensory channels.

Table 1: WHO statistical data of visually impaired users

\begin{tabular}{|l|l|l|l|l|}
\hline $\begin{array}{l}\text { Ages } \\
\text { (Years }\end{array}$ & $\begin{array}{l}\text { Total } \\
\text { Populatio } \\
\text { n } \\
\text { (millions) }\end{array}$ & $\begin{array}{l}\text { Blind } \\
\text { Populatio } \\
\text { n } \\
\text { (millions) }\end{array}$ & $\begin{array}{l}\text { Low } \\
\text { vision } \\
\text { populatio } \\
\text { n } \\
\text { (millions) }\end{array}$ & $\begin{array}{l}\text { Visually } \\
\text { impaired } \\
\text { populatio } \\
\text { n } \\
\text { (millions) }\end{array}$ \\
\hline $0-14$ & 1848.5 & 1.421 & 17.518 & 18.939 \\
\hline $15-49$ & 3548.2 & 5.784 & 74.463 & 80.248 \\
\hline $\begin{array}{l}50 \text { \& } \\
\text { above }\end{array}$ & 1340.8 & 32.16 & 154.043 & 186.203 \\
\hline Total & 6737.5 & $\begin{array}{l}39.365 \\
(0.58 \%)\end{array}$ & $\begin{array}{l}246.024 \\
(3.65 \%)\end{array}$ & $\begin{array}{l}285.389 \\
(4.24 \%)\end{array}$ \\
\hline
\end{tabular}

The first aid for blind people was the long cane developed in 1950 and it is still in use today. It allows the detection of obstacles very close to the user and requires instantaneous reaction when an obstacle is encountered. Dog guide was another mobility aid commonly used by VI people. Both these travel aids help the visually impaired person to avoid obstacles while traveling and are cost-effective. But the major disadvantages of these devices are that they cannot detect head height obstacles and the visually impaired person does not get any information about the type and the dimension of the obstacle. Moreover, the duration required to train the user for using these mobility aids is also long. After 1990s there are few mobility aids designed based on imaging systems. With the development of Bluetooth, radio waves, and RFID tags, few indoor mobility aids were designed using these technologies to help VI people detect door stairs and navigate in closed environments. ROSHNI [9], is an indoor mobility aid developed at IIT Delhi, it is based in infra-red wayfinding technology. The prototype downloads the floor plan of the building and locates and tracks the user inside the building, The algorithm finds the shortest path and gives step by step direction to the user to the desired location.

Published By:

Blue Eyes Intelligence Engineering and Sciences Publication

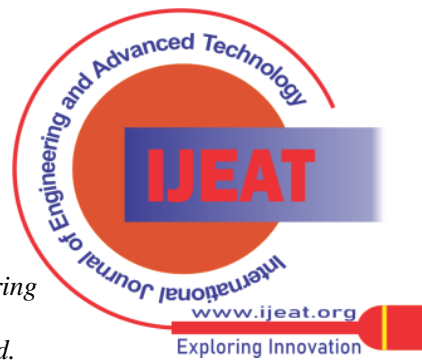


A prototype antenna was developed which is fitted in the door casing. The radiation pattern shape is similar to a doughnut. The user has a receiver which continuously monitors the energy of from the antenna. [10] Kinect based door detection uses a single RGB camera and infrared depth sensor to find if the doors are present in front of the user. In this device the processing is perfoemed on a laptop thus making the system bulky. Guidecane[2] uses 8 ultrasound sensors to find the obstacle in front of the user. Two of these sensors are used to detect stairs. It can detect two upward stairs and two downward stairs. There are prototypes where RFID tags are placed on the floor and each and every step of stairs. The user had a Bluetooth receiver which when pointed in correct direction detects these Bluetooth tags and guides the user to the desired destination.

All the designed prototypes are a bit bulky. Moreover, there is a requirement for separate device for both outdoor travel and indoor travel.

The present paper propose algorithm to detect stairs and doors for indoor navigation. This algorithm is a part prototype designed using stereo cameras that can be also used to detect any obstacle from ground level to head height using depth mapping technique. Section II explains the door detection algorithm. Section III introduces to the steps required to detect stairs. Section IV in brief introduces the mobility aid designed for visually impaired users. Section V discusses the experimental arrangements and data collected during the validation process.

\section{DOOR DETECTION ALGORITHM}

The major characteristic to separate from other obstacles and indoor structures are its edges. Doors are made up of two horizontal and two vertical edges. The angle between the adjacent two edges of the door is always $90^{\circ}$. Perspective projection of the $3 \mathrm{D}$ world on $2 \mathrm{D}$ image plane, makes the angle other than $90^{\circ}$, but the horizontal edges always remain parallel. Since the angle between the edges can vary with the distance between imaging system or user and door, angle feature cannot be used, but horizontal edges and number of vertical edges between them can be used to detect doors. The minimum length of vertical edges and distance between two detected horizontal edges are used as features to distinguish between doors and objects with similar properties like cupboards. The designed algorithm works on the following assumptions:

a. The doors are not made up of transparent material like glass.

b. Vertical edges of the door should always be projected in the image.

c. At least one horizontal edge on the door should be visible.

As shown in figure 1, let C1, C2, C3, C4 be the corners of the doors and X12, X23, X34, X41 be the edges of the door. Let $d$ be the diagonal distance of the image. The ratio of the edge length and diagonal distance $\mathrm{D}$ is given by equation 2 . The angle of the edge is given by equation 3 .

$$
S_{i j}=\frac{\left|\left(X_{i}-X_{j}\right)^{2}+\left(Y_{i}-Y_{j}\right)^{2}\right|}{D}
$$

The algorithm developed for detecting doors from the captures images is as follows:

a) Apply Gaussian filter to the left stereo image. This will help reduce unwanted edge.

b) Apply the canny edge detector to convert a grayscale image into a binary image. The locations where edges are present are marked with pixel intensity 255 and rest are marked with intensity 0 .

c) Apply Hough line transform. This algorithm helps to detect only straight-line edges and no curved edges are detected.

d) Remove the unwanted vertical edges by applying the limits on the minimum distance between edges.

e) Remove unwanted horizontal edges by applying the limit on the maximum length of horizontal edges.

f) Check if there is at least one horizontal edge between two vertical edges. If yes, then that is a probable door. If no horizontal edge, then that cannot be a door.

g) Calculate the midpoint of the detected door. The ation of the user is informed to the user using auditory feedback.

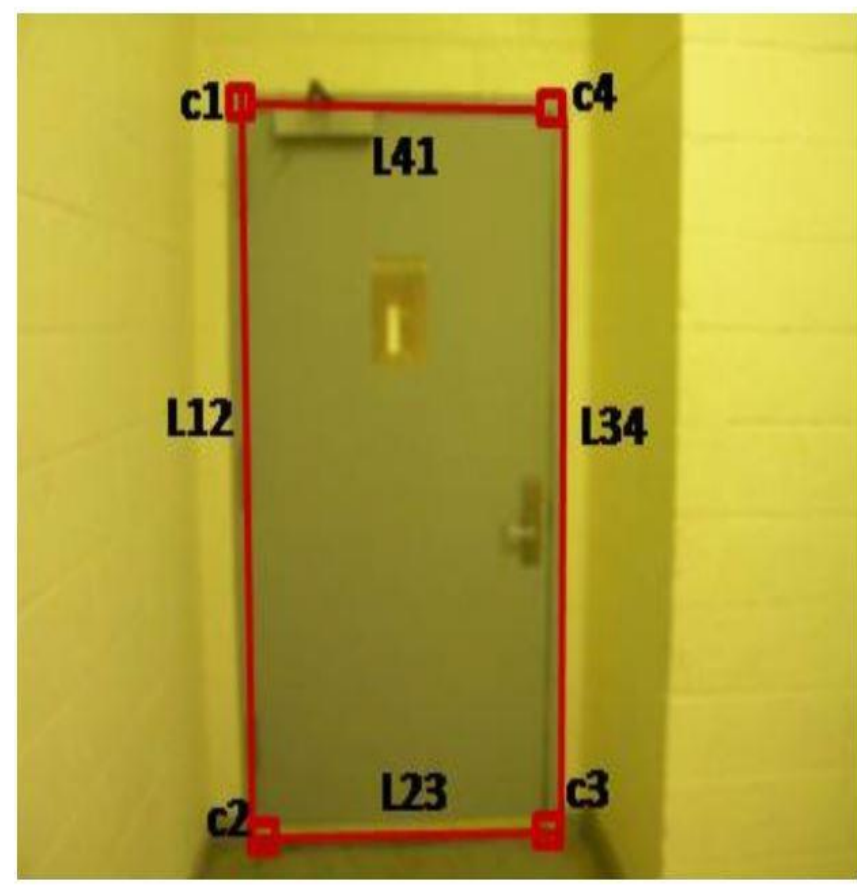

Figure 1: Parameters for door analysis$$
\text { - Copyrigh: All rights reserved. }
$$ 


\section{STAIR DETECTION ALGORITHM}

Stairs can be distinguished from other obstacles by its unique property that all the edges of stairs are parallel. As shown in figure 3 , stairs are characterized by one more feature, they have the parallel edges bounded between two diagonal lines or one diagonal edge

These two features along with an additional feature of the distance between two parallel lines can help detect stairs and distinguish stairs from other obstacles with similar features like the bookshelf. After studying 50 images of stairs and 35 images of bookshelf, it was observed that if the distance between two parallel lines is more than 50 pixels then they cannot be considered as stairs. These measurements were done when the stereo system was at a distance of 2.5 to 3.0 meters from the stairs or bookshelf.

The algorithm to detect indoor stairs is as follows:

a) Apply Gaussian filter to the left stereo image. This will help reduce unwanted edges.

b) Apply canny edge detector to convert a grayscale image into a binary image. The locations where edges are present are marked with pixel intensity 255 and rest are marked with intensity 0.

c) Apply Hough line transform. This algorithm helps to detect only straight-line edges and no curved edges are detected.

d) Remove the unwanted vertical edges by applying the limits on the angle of the vertical lines.

e) Remove unwanted horizontal edges by applying the limit on the angle of horizontal edges.

f) Check if horizontal lines are more than the threshold and distance between the horizontal lines is not more than 50 pixels

g) If the condition of the number of horizontal lines, space between horizontal lines, and if one of the diagonal edges are present then the image contains stairs. The midpoint of the smallest edge of the staircase is calculated.

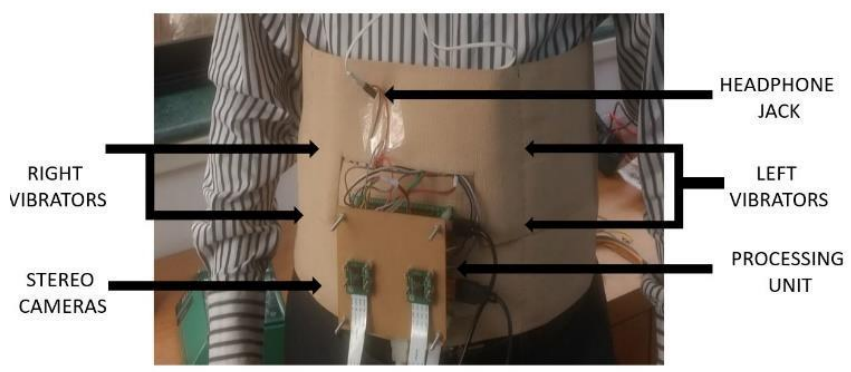

Figure 2 : Designed mobility aid prototype
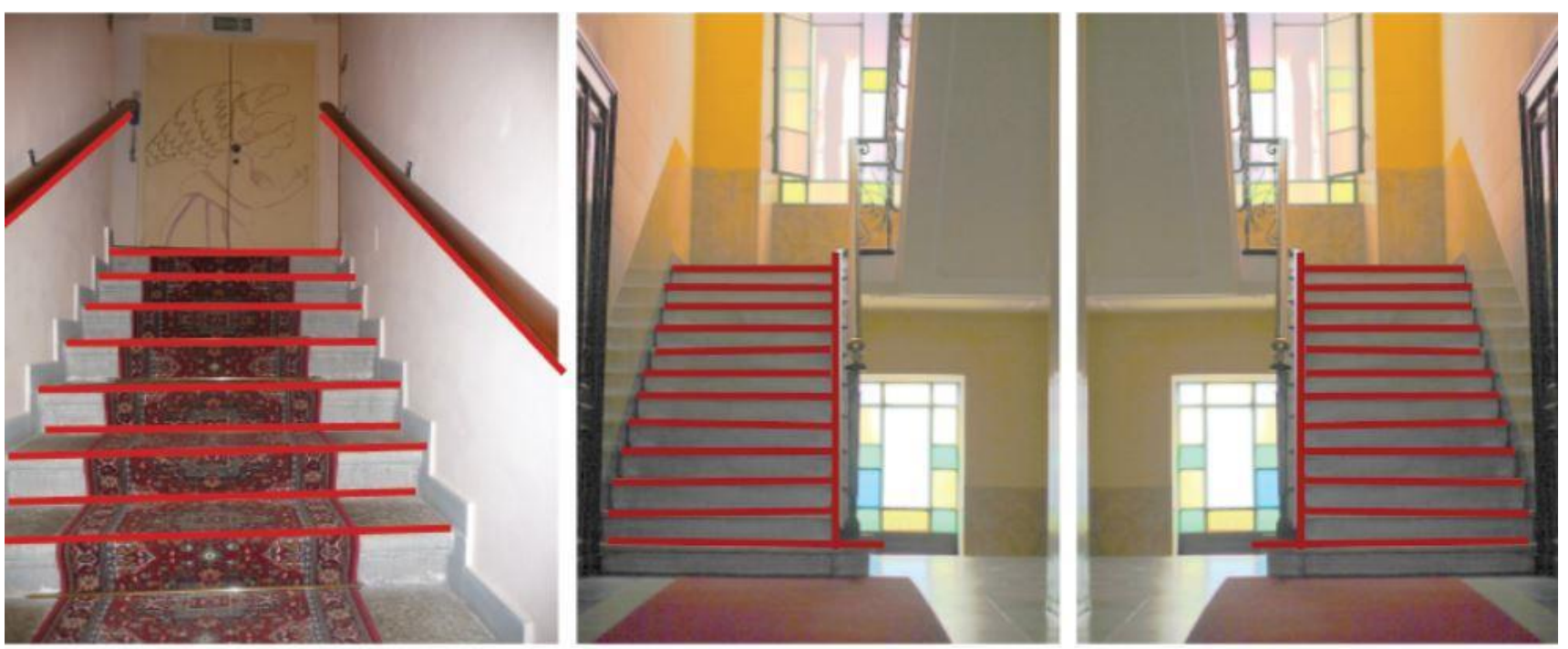

Figure 3: Criteria considered for stair detection

\section{HARDWARE PROTOTYPE}

The hardware of the designed prototype is as shown in the figure 2

This prototype is placed around the waist using orthopedic belt. It consists of stereo cameras, placed in front to capture the area in front of the visually impaired user. The customed made board analyzes the captures images at a rate of 1 frame per second. The processing is done on ARM quad core processor with $8 \mathrm{~GB}$ of SSD and 1GB of RAM. This device is battery operated and has an in-built lithium-ion battery that can run the device for 16 hours at full capacity. There are four vibrators' paces on back side of the belt to inform user about path to be taken to avoid the obstacles. The board also has mono headphone jack to provide auditory feedback to the user. Door and stair information is provided to the user using auditory feedback.
Table 2 indicates the auditory feedback given to the user in regard to doors and stairs information

Table 2: Auditory feedback given to user

\begin{tabular}{|rl|}
\hline \multicolumn{1}{|c|}{ Auditory Feedback } \\
\hline 1. & Door Detected on your left \\
\hline 2. & Door detected on your right \\
\hline 3. & Door straight in front of you \\
\hline 4. & Stairs on your left \\
\hline 5. & Stairs on your right \\
\hline 6. & Stairs in front of you \\
\hline
\end{tabular}

Published By:

Blue Eyes Intelligence Engineering and Sciences Publication

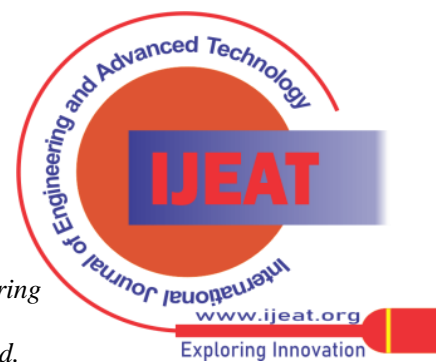




\section{EXPERIMENTAL ARRANGEMENT AND RESULTS}

Door detection and stair detection algorithm was written in $\mathrm{C}++$ and OpenCV modules were used to extract feature important for classification of stairs and doors from non-stairs and non-door structures.

To determine the limits required to distinguish stairs and doors from other indoor structures, 100 images of various indoor structures were captured by the developed prototype. After comparing these images, it was observed that when the user is standing at a distance of 3.0 meters from the door, height $_{\text {low }}=500$ pixels and height high $=620$ pixels. Similarly, it was observed that width ${ }_{\text {low }}=250$ pixels and width $_{\text {high }}=320$ pixels. Similarly, for stairs it was also concluded that the diagonal edges of stairs have an angle between 0.4 radians to 0.6 radians or between 2.8 radians to 3 radians.

The designed prototype and algorithm were tested on 50 visually impaired users. The validation was broken down in two stages:

1. Training stage:

In this stage the users were asked to wear the device and they were trained to react to the auditory and vibratory stimulus provided that would help them to react to obstacles and indoor structures like doors and stairs. During this stage the time required to train the user was recorded.

2. Validation stage:

In this stage the users were asked to walk in controlled indoor environment and the response to various indoor structures were recorded. They were exposed to varying number of doors and stairs. Arrangement of indoor navigation is as shown in the figure 5.
During this stage the percentage of accurately recognizing doors and stairs and percentage of correctly distinguishing the doors and stairs from other indoor structures was calculated.

The experiment was repeated three times and the average of the results obtained for each user was recorded.

Following figure indicates the percentage of correctly the algorithm detected door.

From the figure 4 it is clear that average accuracy of correctly detecting doors in indoor environment is around $75 \%$.

Similarly figure 6 shows the accuracy of correctly detecting stairs and giving correct feedback to the user. It was observed that the on an average 85 percent of times users to detect the exact location of stairs

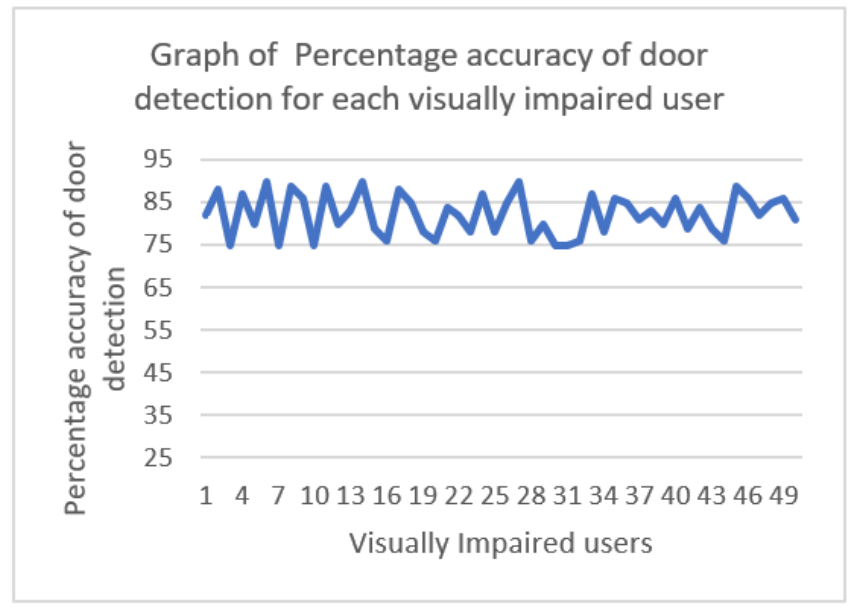

Figure 4: Graph of Percentage accuracy of door detection

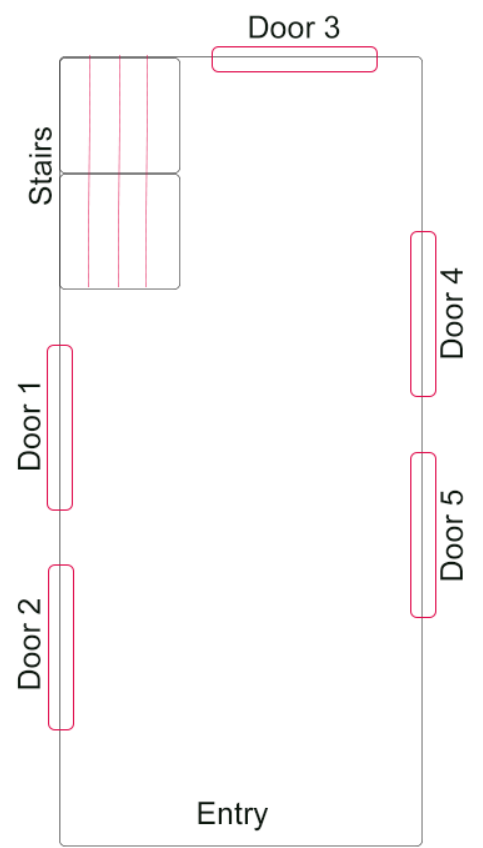

(A)

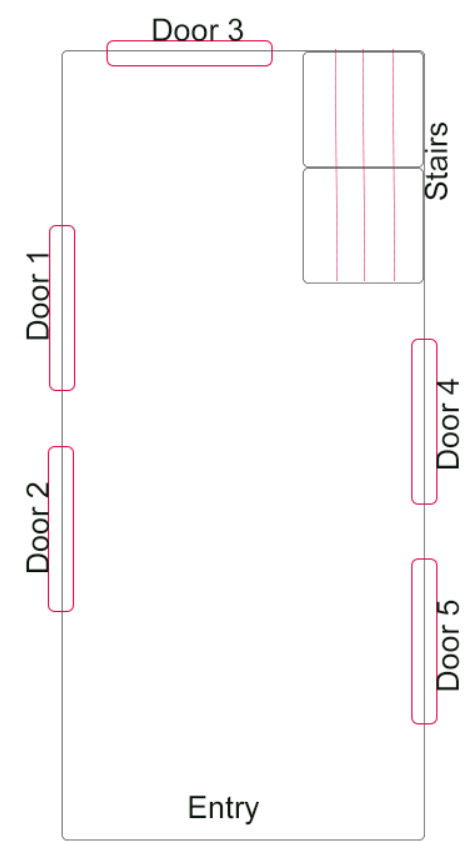

(B)

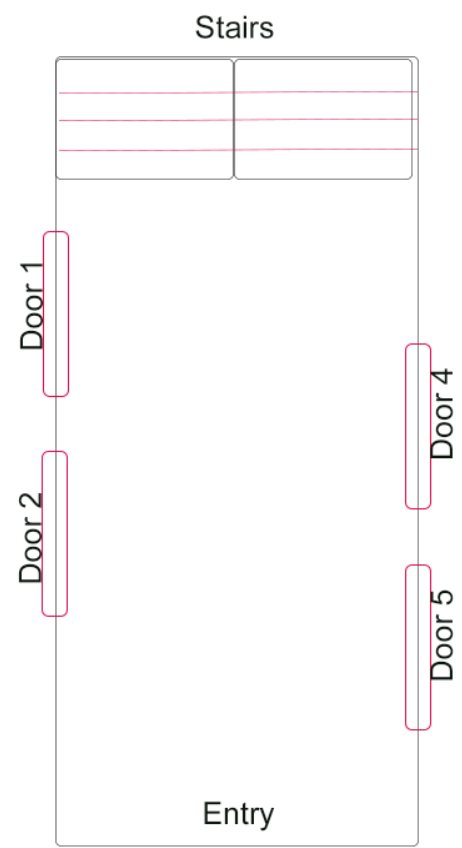

(C)

Figure 5: Experimental arrangement for validation process

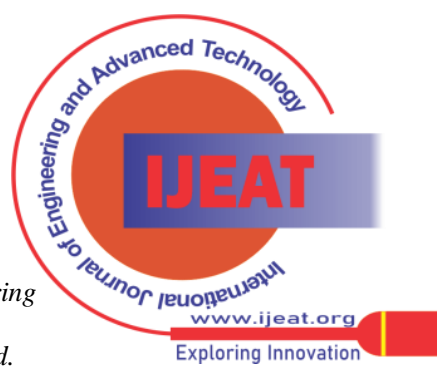




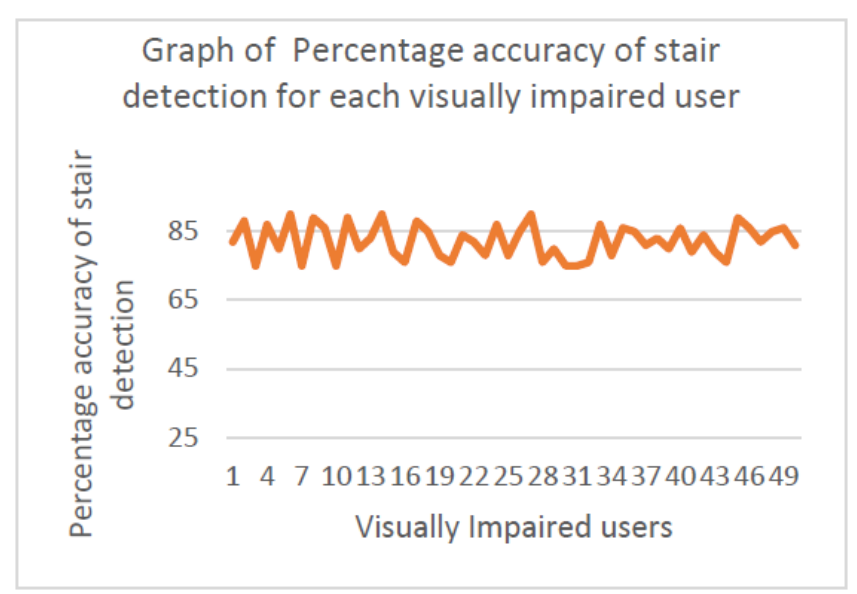

Figure 6: Graph of percentage accuracy of stair detection

\section{CONCLUSION}

In this paper, we have presented developed mobility aid and door and stair detection algorithm that can help any visually impaired user to navigate in indoor environments. The total processing time required for capturing stereo images, preprocessing those images, extracting information of stairs and doors and relaying this information in form of audio to user takes about 0.15 to 0.2 seconds. The accuracy of detection of doors is around 75 percent and for stairs is around 85 percent. The uses were able to use the designed prototype along with a white cane to navigate in indoor environments with ease. As we have used a mono headphone feedback system, it does not affect the information that visually impaired user generates from natural auditory cues like the sound beeps installed in the closed premises to guide them. There is a vast scope of improvement that can be done in the design. With the use of machine learning, more features of doors and stairs can be extracted and analyzed to increase the accuracy of the detection. Using the depth map generated by the stereo images, we can also detect whether the stairs are upward stairs or downward stairs.

\section{ACKNOWLEDGMENT}

The authors would like to thank the electronics department at Veermata Jijabai Technological Institute for their support in the development of the mobility aid prototype. Authors would also like to thank members and students of National Association of blind and rehabilitation center, Mumbai, NAB school for the blind, Nashik for their co-operation during the validation process of the prototype

\section{REFERENCES}

1. Global data on visually impairment. World Health Organization, 2010

2. L. Dunai, I. Lengua Lengua, I. Tortajada, and F. Brusola Simon, "Obstacle detectors for visually impaired people," in International Conference on Optimization of Electrical and Electronic Equipment (OPTIM), pp. 809-816, May 2014

3. R. Jafri, S. A. Ali, H. R. Arabnia, and S. Fatima, "Computer vision-based object recognition for the visually impaired in an indoors environment: a survey," The Visual Computer, vol. 30, no. 11, pp. 1197-1222, 2014

4. X. Lu and R. Manduchi, "Detection and localization of curbs and stairways using stereo vision," in Proceedings of the 2005 IEEE International Conference on Robotics and Automation, pp. 4648-4654, April 2005.

5. S. Wang and H. Wang, "2d staircase detection using real AdaBoost," in 2009 7th International Conference on Information, Communications and Signal Processing (ICICS), pp. 1-5, Dec 2009.

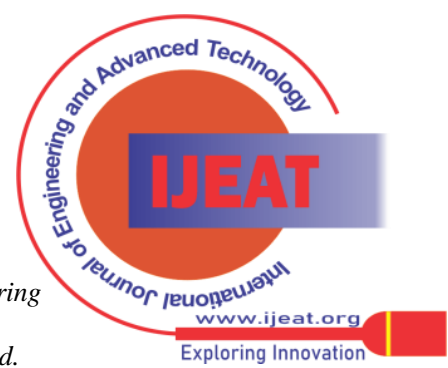

Shrugal Varde, received his Bachelor of Engineering degree in Electronics from the University of Mumbai, India. He received his Master of Technology degree in Electronics from Veermata Jijabai Technologica Institute (VJTI) of Mumbai, India. He is currently pursuing his Ph.D. degree in the Electronic Engineering Department at VJTI. His project incorporates the development of a computer vision based portable mobility aid for the visually impaired. He has authored and co-authored research papers published in several international journals and IEEE conference proceedings. His research interests include image processing, compute vision, machine learning, human-computer interactions and embedded

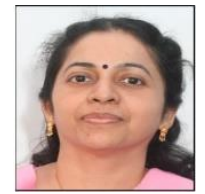

Dr. M.S. Panse, received her Ph. D degree in electronic Engineering from Veermata Jijabai Technological Institute, Mumbai, India. She is currently professor in electronic department at Veermata Jijabai technological Institute (VJTI), fellow member of Indian Society of Engineers society of India She is author and co-author on papers published extensively in International Journals, book chapters and conference proceedings. Her current research interest includes Biomedical Instrumentation, Medical Electronics, Virtual Instrumentation, mobility aids for visually impaired and sensors. 